\section{Pillen-Prophylaxe vor Katzenkontakt}

\author{
Antihistaminika reduzieren effektiv die Symptome einer aller- \\ gischen Rhinokonjunktivitis. Doch warum eigentlich warten, bis \\ Beschwerden auftreten, wenn eine prophylaktische Einnahme die \\ Rhinitis frühzeitig in Schach halten könnte?
}

드. in US-Forscherteam ging der Frage nach, ob die prophylaktische Gabe des $\mathrm{H}_{1}$-Rezeptorantagonisten Fexofenadin vor einer Katzenhaarexposition die Symptome bei entsprechend allergischen Patienten verringern kann. Dazu erhielten 66 überwiegend weibliche Probanden randomisiert und doppelblind jeweils 1,5 Stunden vor einem Provokationstest entweder $180 \mathrm{mg}$ Fexofenadinhydrochlorid oder Plazebo. Mindestens 14 Tage später wurde der Versuch mit Verum und Plazebo vertauscht wiederholt. Die Probanden, deren Katzenhaar- sensibilisierung durch Pricktest nachgewiesen worden war, mussten sich zur Provokation bis zu einer Stunde in einem Raum aufhalten, in dem zuvor Katzen gewesen waren. Alle fünf Minuten dokumentierten sie auf einer fünfstufigen Skala die Schwere der Symptome Rhinorrhö, Juckreiz in Nase, Gaumen und Rachen, Niesen sowie Juckreiz, Tränen und Rötung der Augen. Aus diesen Angaben wurde ein Symptomscore errechnet.

Insgesamt 63 Patienten schlossen die Studie ab. Unter der Prämedikation mit Fexofenadin nahm der Symptom- score nach 30 Minuten Katzenhaarexposition signifikant weniger $\mathrm{zu}$ als unter Plazebo $(\mathrm{p}=0,03)$. Für die meisten Einzelsymptome traf dies ebenfalls zu, der nasale Flow blieb allerdings die ganze Beobachtungszeit über unverändert. Die berichteten unerwünschten Ereignisse waren mild bis mäßig und unter Verum und Plazebo vergleichbar häufig.

Fazit: Den Ergebnissen dieser - bezüglich der Allergendosis nicht quantifizierbaren - Studie zufolge kann die prophylaktische Einnahme von Fexofenadin vor Katzenkontakt die Symptome einer Katzenhaarallergie signifikant verringern. Sinnvoll ist dies sicher nur bei einer gelegentlichen Exposition.

Berkowitz RB et al. Efficacy of fexofenadine in the prophylactic control of cat allergen-induced allergic rhinitis. Ann Allergy Asthma Immunol 2006; 96: 327-33

\title{
Bei einer Nuss bleibt es selten
}

\section{Typ-I-Reaktionen auf Nüsse sind schon bei Kindern sehr häufig. Eine Querschnittsstudie versuchte dem natürlichen Verlauf einer Nuss- allergie bei Kindern im Alter zwischen zwei und zehn Jahren auf die Spur zu kommen.}

K onsekutiv wurden 784 Kinder, die mit Symptomen nach einem gesicherten Konsum von Nüssen eine Allergieambulanz im britischen Cambridge aufgesucht hatten, untersucht. Es wurde das spezifische IgE gegen verschiedene Nussarten bestimmt, weiter wurden Pricktests durchgeführt. Am häufigsten war bei 68\% der Kinder eine klinisch manifeste Allergie gegen die Hülsenfrucht Erdnuss, gefolgt von Allergien gegen Paranuss (12\%), Haselnuss (6\%), Walnuss (3\%) und Mandel (2\%).

Von den Kindern, die bereits im Alter von zwei Jahren erstmals vorgestellt wurden, war der Großteil nur gegen eine Nussart allergisch, lediglich $2 \%$ hatten mehrere klinisch relevante Nussallergien.

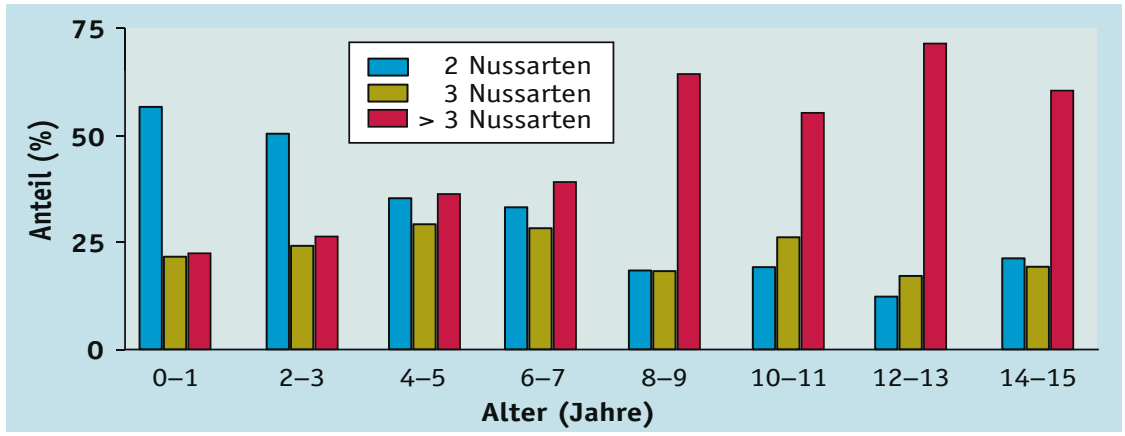

Anteil mehrfacher Sensibilisierungen in Abhängigkeit vom Alter bei Erstvorstellung bei 531 Kindern mit Nussallergie
Eine mehrfache Sensibilisierung konnte anhand des spezifischen IgE aber schon bei $19 \%$ dokumentiert werden. Dieser hohe Prozentsatz ist verblüffend, deutet er doch auf eine Sensibilisierung in einer sehr frühen Lebensphase, vielleicht bereits im Mutterleib, hin.

Je älter die Kinder dann bei Erstvostellung in der Klinik waren, mit umso mehr Nusssorten waren sie vor der ersten allergischen Reaktion bereits in Kontakt gekommen. Parallel dazu nahm auch der Anteil der Kinder mit mehrfacher Sensibilisierung zu: Im Alter von zehn Jahren war dies schon bei 86\% der Patienten der Fall, unter Allergien gegen mehrere Nussarten litten bereits $47 \%$.

Fazit: Sensibilisierungen gegen Baumnüsse oder Erdnuss sind bei Kindern häufig und kommen sogar schon im Säuglingsalter vor. Die Betroffenen entwickeln im Verlauf der Jahre oft weitere Sensibilisierungen und Allergien gegen Nüsse.

Clark AT et al. The development and progression of allergy to multiple nuts at different ages. Pediatr Allergy Immunol 2005; 16: 507-11 